\title{
ON MONOIDS OF FINITE REAL CHARACTER
}

\author{
ULRICH KRAUSE
}

(Communicated by Louis J. Ratliff, Jr.)

\begin{abstract}
The property of Krull domains, to be determined already by its essential valuations, is extended to a class of monoids of finite real character containing Krull monoids. This is applied to construct a divisor theory for such monoids and also to characterize Krull domains purely multiplicatively as the integral domains for which the multiplicative monoid is a Krull monoid.
\end{abstract}

\section{INTRODUCTION}

Krull monoids, which are defined analogously to Krull domains, have been studied by Chouinard II [2] and Gilmer [3]. In [2] it is observed already, that the property of Krull domains that the essential valuations form the smallest defining family of discrete valuations carries over to the analogous result for Krull monoids. (Cf. Corollary 1 below and the remarks thereafter.) In the present paper we extend this to more general monoids including, besides Krull monoids, rational generalized Krull monoids and also nonalgebraic objects like the polyhedral cones of convex analysis. The proof is direct and simple, requiring not more effort than a concise proof in the case of Krull domains like that given in [7]. The main step in the proof is the description of essential states on preordered Abelian groups as given in $\S 2$. Our theorem yields in particular an easy construction of a divisor theory for Krull monoids and more general monoids. As a further application we obtain in $\S 3$ a purely multiplicative characterization of Krull domains which states that an integral domain is a Krull domain if and only if its multiplicative monoid is a Krull monoid.

By a monoid we mean a commutative and cancellative semigroup $S$ with unit. Since $S$ can be embedded into its quotient group $G$, we may, equivalently, consider an additively written Abelian group $G$ with a monoid or cone $S$ in $G$, i.e., a subset $S \subset G$ with $O \in S$ and $S+S \subset S$, which generates $G$, i.e., $G=S-S$. Thereby for subsets $A, B \subset G, A \pm B=\{a \pm b \mid a \in A, b \in B\}$. We can also speak of a preordered group $(G, S)$. The preorder being defined by

Received by the editors October 14, 1987 and, in revised form July 20, 1988.

1980 Mathematics Subject Classification (1985 Revision). Primary 13A18, 20M25; Secondary 06F20, 13 F 30.

Key words and phrases. Partially ordered groups, essential valuations, divisor theory, Krull monoids, Krull domains. 
$x \leq y$ iff $y-x \in S$, is directed, i.e., every element is a difference of two nonnegative elements. (Cf. [4] on these matters.) We shall employ this language in the following, since not only multiplicative monoids of integral domains but also certain cones in the additive group of real vector spaces fit into our framework. A homomorphism $f$ of $G$ into the additive group of real umbers $\mathbb{R}$ which is not identically zero $(f \not \equiv 0)$ and which maps $S$ into the cone $\mathbb{R}_{+}$of non-negative real numbers is said to be a state on $(G, S)$. (The notion of a state used in [4] is more special in that it requires additionally $G$ to possess an order unit.)

\section{ESSENTIAL STATES ON PARTIALLY ORDERED GROUPS}

A state $f$ on a preordered group $(G, S)$ is said to be an essential state on $(G, S)$, if for any $x, y \in G$ there exists a $z \in G$ such that

$$
x \leq z, \quad y \leq z \quad \text { and } f(z)=\max \{f(x), f(y)\} .
$$

An essential state may be characterized also by looking at the cone $P=f^{-1}\left(\mathbb{R}_{+}\right)$ defined by the state $f$. One verifies easily that a state $f$ on a directed preordered group $(G, S)$ is essential if and only if the cone $P$ defined by $f$ satisfies the equation $P=S-(S \cap P \cap(-P))$. Read multiplicatively, this equation is more akin to commutative algebra and serves in [3, p. 201] to define an essential valuation on the quotient group of a monoid. Because it is easier to work with we shall stick however to the above given definition of an essential state. Our aim is to identify the essential states for the type of monoids defined below.

A nonempty family $F$ of group homomorphisms $f: G \rightarrow \mathbb{R}, f \not \equiv 0$, is a family of compact real character, if all the sets $\{f \in F \mid f(x) \neq 0\}$ for $x \in G$ have compact closure in $F$ for the topology induced on $F \subset \mathbb{R}^{G}$ by the product topology of $\mathbb{R}^{G}$. A special case is a family of finite real character where the sets $\{f \in F \mid f(x) \neq 0\}$ for $x \in G$ are finite sets. Since all homomorphisms under consideration are into the real numbers $\mathbb{R}$, the addendum "real" will be omitted in what follows.

A monoid, or cone, $S$ is defined by a family $F$ of homomorphisms if $S=$ $\cap\left\{f^{-1}\left(\mathbb{R}_{+}\right) \mid f \in F\right\} . S$ is a monoid of compact (finite) character if $S$ is defined by some family of compact (finite) character. A homomorphism $f: G \rightarrow \mathbf{R}$ is rational, if the image $f(G)$ is contained in the additive group $\mathbb{Q}$ of rational numbers, and $f$ is discrete if $f(G) \subset \mathbb{Z}, \mathbb{Z}$ the additive group of the rational integers. For a family $F$ of homomorphisms, $f \in F$ is said to be adapted, whenever for any $x, y \in G$ and any $r, s \in f(G)$ there exist $z \in G$ and $0 \neq t \in \mathbb{R}$, such that $\operatorname{rg}(x)+\operatorname{sg}(y)=\operatorname{tg}(z)$ for all $g \in F$. Obviously, any discrete or rational homomorphism is adapted for any family to which it belongs. Another, completely different, example is given by a real vector space $G$ and some family of vector space homomorphisms into the reals.

For two families $F$ and $F^{*}$ of states, the family $F$ is said to be smaller than $F^{*}$ if for each $f \in F$ there is some $r>0$ such that $r f \in F^{*}$. 
Lemma. Let $(G, S)$ be a directed preordered group.

(1) If $S$ is a monoid of compact character with defining family $F$, then the set of essential states on $(G, S)$ is smaller than $F$.

(2) If $S$ is a monoid of finite character with defining family $F$, then every adapted $f \in F$ for which $F \backslash\{f\}$ does not define $S$ must be an essential state on $(G, S)$.

Proof. (1) First we show that to every state $f$ on $(G, S)$ there exists some $g \in F$ such that $S \cap f^{-1}(0) \subset g^{-1}\left(\mathbf{R}_{-}\right), \mathbf{R}_{-}=\{x \in \mathbf{R} \mid x \leq 0\}$. Suppose this is not the case, that is to every $g \in F$ there exists some $y_{g} \in S \cap f^{-1}(0)$ with $g\left(y_{g}\right)>0$. Consider for arbitrary but fixed $x \in G$ the set $M=\{g \in F \mid g(x)<$ $0\}$.

By assumption, $M$ has compact closure in $F$. For each $g \in F$ there exists some $n_{g} \in \mathrm{N}$ such that $g\left(n_{g} y_{g}+x\right)>0$. The family of all the sets $U_{g}=\left\{h \in F \mid h\left(n_{g} y_{g}+x\right)>0\right\}$ is an open cover of the closure of $M$ and must contain a finite subcover, say $\left\{U_{g}\right\}_{g \in E}$. Hence to $h \in M$ there exists some $k=k(h) \in E$ such that $h\left(n_{k} y_{k}+x\right)>0$. If $y=\sum_{g \in E} n_{g} y_{g}$, then $h(x+y)=$ $\sum_{g \neq k} h\left(n_{g} y_{g}\right)+h\left(n_{k} y_{k}+x\right)>0$. Of course, if $h \in F \backslash M$, then $h(x) \geq 0$ and $h(x+y) \geq 0$ because of $y \in S$. Thus $h(x+y) \geq 0$ for all $h \in F$ and $x+y \in S$. Because of $y_{g} \in f^{-1}(0)$ we must have $f(x)=f(x)+f(y)=f(x+y) \geq 0$. That is, $f$ must be identically zero, which contradicts the definition of a state. This shows $S \cap f^{-1}(0) \subset g^{-1}\left(\mathbf{R}_{-}\right)$for some $g \in F$. Assuming $f$ to be an essential state it follows that $f^{-1}\left(\mathbf{R}_{-}\right) \subset g^{-1}\left(\mathbf{R}_{-}\right)$. For this let $f(x) \leq 0$. There exists $z \geq x, z \geq 0$ such that $f(z)=\max \{f(x), f(0)\}=0$. From $z \in S \cap f^{-1}(0)$ it follows that $0 \geq g(z) \geq g(x)$. Furthermore, there is some $a \in G$ with $s=g(a)>0$, because of $g \not \equiv 0$. Hence $t=f(a)>0$ by $f^{-1}\left(\mathbf{R}_{-}\right) \subset g^{-1}\left(\mathbf{R}_{-}\right)$. The latter inclusion carries over to $f_{1}=(1 / t) f, g_{1}=$ $(1 / s) g$, that is $f_{1}^{-1}\left(\mathbf{R}_{-}\right) \subset g_{1}^{-1}\left(\mathbf{R}_{-}\right)$. Suppose $f_{1} \neq g_{1}$, i.e., $g_{1}(x)<f_{1}(x)$ for some $x \in G$. Then there are $m, n \in \mathbf{Z}, n>0$, with $g_{1}(x)<m / n<f_{1}(x)$. It follows $0<m-n g_{1}(x)=g_{1}(m a-n x)$ and $0>m-n f_{1}(x)=f_{1}(m a-n x)$, which is a contradiction. Therefore we must have $f_{1} \equiv g_{1}$, that is $f=r g$ with $r=t / s>0, g \in F$.

(2) Let $x, y \in G$ and, without restriction, $f(x) \leq f(y)$. Then $f \notin M=$ $\{g \in F \mid g(y)<g(x)\}$ and $M$ is a finite set by assumption. Since $G=S-S$, there exists for each $g \in F$ some $u_{g} \in S$ with $g\left(u_{g}\right)>0$. If $u=\sum_{g \in M} u_{g}$, then $u \in S$ and $g(u)>0$ for all $g \in M$. By assumption, $F \backslash\{f\}$ does not define $S$ and therefore there is some $v \in G$ such that $g(v) \geq 0$ for all $g \in$ $F \backslash\{f\}$ but $f(v)<0$. Since $f$ is adapted, we can find to $u, v \in G, r=f(-v)$ and $s=f(u)$ some $t>0$ and some $w \in G$ such that $r g(u)+s g(v)=\operatorname{tg}(w)$ for all $g \in F$. Setting $g=f$, we obtain $f(w)=0$. If $g \in F \backslash\{f\}$, then $g(v) \geq 0$ and hence $g(w) \geq 0$ because of $u \in S$. Thus $w \in S$. Furthermore, $g(u)>0$ and $g(v) \geq 0$ for $g \in M$ and hence $g(w)>0$ for $g \in M$. Therefore we can find $n \in \mathbb{N}$ such that $n g(w) \geq g(x-y)$ for all $g \in M$. Define 
$z=y+n w$. Obviously $z \geq y$ and $g(z-x) \geq 0$ for $g \notin M$. For $g \in M$ $g(z-x)=g(y-x)+n g(w) \geq 0$ by definition of $n$. Hence $g(z-x) \geq 0$ for all $g \in F$, that is $z \geq x$. Finally, $f(z)=f(y)+n f(w)=f(y)$ and $f(z)=\max \{f(x), f(y)\}$. Q.E.D.

To illustrate the notion of an essential state we discuss some examples.

Examples. (1) Simple examples of monoids of finite character are $\mathbb{N}=\mathbb{Z}_{+} \backslash\{0\}$ $=\{1,2,3, \ldots\}$ or the Hilbert monoid $S=\{4 n+1 \mid n=0,1,2, \ldots\}$, both with respect to multiplication. (Cf. [6, p. 28] for a discussion of the Hilbert monoid.) As every factorial monoid (cf. [3, p. 57] for the definition), the monoid $\mathbf{N}$ is defined by its essential states, which, as in valuation theory, are determined by the prime elements. The Hilbert monoid $S$ is not factorial, but this monoid too is defined by its essential states, which are obtained by restricting those on $\left(\mathbb{Q}_{+} \backslash\{0\}, \mathbb{N}\right)$ to the quotient group of $S$. The additive monoid $\mathbb{Z}_{+}$is defined by the identity which is (up to a positive scalar) the only state and which is essential. The additive monoid $\mathbb{Z}_{+} \backslash\{1\}$ however cannot be defined by a family of states. The only state (up to a positive scalar) is the identity which is not essential.

(2) A simple example of a monoid of compact, but not necessarily finite, character, is the following one. The example may be considered some generalization of the notion of a factorial monoid. Let $S$ be the cone of all non-negative realvalued continuous functions on some topological space $T$ which have compact support. Take $S$ with pointwise addition and let $G=S-S$. If $\varepsilon_{t}$ denotes evaluation at $t \in T$, i.e., $\varepsilon_{t}(x)=x(t)$ for $x \in G$, then $F=\left\{\varepsilon_{t} \mid t \in T\right\}$ is a family of compact character defining $S$. Obviously, any $\varepsilon_{t}$ provides an essential state on $(G, S)$. By part (1) of the lemma, these are-up to some positive scalar-all essential states. The special case where $T$ is equipped with the discrete topology and the functions are $\mathbb{Z}$-valued corresponds to the case of factorial monoids (with $T$ being the set of primes).

(3) In the above example the notion of an essential state coincides with that of an extremal state. Thereby a state $f$ on $(G, S)$ is extremal if from $g(x) \leq f(x)$ for some state $g$ and all $x \in S$ it follows that $g=r f$ for some $r>0$. Part (1) in the proof of the lemma shows that an essential state must be necessarily extremal. The reverse implication is not true in general. Consider for example the ice cream cone

$$
S=\left\{x=\left(x_{1}, x_{2}, x_{3}\right) \in \mathbf{R}^{3} \mid x_{2}^{2}+x_{3}^{2} \leq 3 x_{1}^{2}, 0 \leq x_{1}\right\}
$$

with addition. By $f(x)=x_{1}-(1 / \sqrt{3}) x_{2}$ an extremal state $f$ on $\left(\mathbb{R}^{3}, S\right)$ is defined. However, taking $x=(1 / \sqrt{3}, 1,1)$ there is no $z \in S$ such that $z-x \in S$ and $f(z)=0$. Hence $f$ is not essential. The above-mentioned reverse implication holds under certain assumptions, e.g., for discrete states. (For the special case of interpolation groups an analysis of extremal states may be found in [4, pp. 110,199]. There some property for states is considered which is similar to, but different from, that defining an essential state.) 
The following theorem is a consequence of the lemma.

Theorem. Let $S$ be a monoid of finite character with a defining family consisting only of adapted states. Then among the families of compact character defining $S$ there is a smallest one, and that consists of essential states on $(G, S)$.

Proof. By part (1) of the lemma it suffices to find some family of essential states which is of compact character and defines $S$. First, we show that the family $E$ of all essential states defines $S$. Assuming $f(x) \geq 0$ for all $f \in E$ we have to show $x \in S$. Let $F$ be a defining family for $S, F$ of finite character and consisting of adapted states. The set $M=\{f \in F \mid f(x)<0\}$ is finite with $M \cap E=\varnothing$. From part (2) of the lemma it follows that for any $f \in M$ the family $F \backslash\{f\}$ defines $S$. The family $F \backslash\{f\}$ satisfies the same assumptions as $F$ and therefore $F \backslash\{f, g\}$ defines $S$ for any $g \in M \backslash\{f\}$. Finiteness of $M$ yields that $F \backslash M$ defines $S$. Of course, $f(x) \geq 0$ for all $f \in F \backslash M$ and hence $x \in S$. Since $E$ itself is not of compact character we select for each $f \in F$ just one $\bar{f}$ from the ray $\{r f \mid r>0\}$. Then $\bar{E}=\{\bar{f} \mid f \in E\}$ is a family of essential states which too defines $S$. Consider for fixed $x \in G$ the sets $M=\{\bar{f} \in \bar{E} \mid \bar{f}(x) \neq 0\}$ and $N=\{h \in F \mid h(x) \neq 0\}$. For $\bar{f} \in M$ there exists, by part (1) of the lemma, some $r>0$ such that $r \bar{f} \in N$. If $\bar{g} \in M$ and $\bar{g} \neq \bar{f}$, then for some $s>0 s \bar{g} \in N$ and $r \bar{f} \neq s \bar{g}$. Since $N$ is finite by assumption, $M$ must be finite too. Thus $\bar{E}$ is of finite character. Q.E.D.

The conclusion of the theorem is no longer true if the monoid $S$ is only required to be of compact character. Consider the ice cream cone $S$ discussed before. Let $F$ be the set of linear functionals $f$ on $\mathbb{R}^{3}$ satisfying $S \subset f^{-1}\left(\mathbb{R}_{+}\right)$ and $\|f\| \leq 1$. Then $F$ is a family of compact-but not finite-character consisting of adapted states. By the Hahn-Banach theorem $S$ is defined by $F$. However, there are no essential states on $\left(\mathbb{R}^{3}, S\right)$.

$A$ monoid of finite discrete (rational, adapted) character is a monoid defined by a family of finite character which consists of discrete (rational, adapted) states. A monoid of finite discrete (rational) character will also be called a Krull monoid (rational generalized Krull monoid). (Cf . [2, p. 1460], [3, p. 190].) The theorem yields immediately the following corollary.

Corollary 1. For every Krull monoid (rational generalized Krull monoid) there exists a smallest defining family of finite character and that consists of discrete (rational) essential states.

Remarks. (1) Obviously, the multiplicative semigroup of a Krull domain (rational generalized Krull domain) is a Krull monoid (rational generalized Krull monoid). Hence Corollary 1 implies the well-known corresponding results for Krull domains.

(2) For the case of Krull monoids, Corollary 1 has been observed by Chouinard II [2, p. 1461]. There it is pointed out that the arguments by Bourbaki [1] which show that the family of essential valuations of a Krull domain is the smallest defining family can be modified to give a similar result for a 
Krull monoid. By a different approach Gilmer [3, p. 201] obtains for Krull monoids Corollary 1 from the corresponding result for Krull domains. Applying in the proof a result about semigroup rings, Gilmer assumed that for each $0 \neq g \in G=S-S$ there are only finitely many integers $n$ for which $n x=g$ is solvable in $G$. Corollary 1, however, holds without that assumption.

The theorem also supplies an easy access to a divisor theory for Krull monoids and more general monoids. Let $S$ be any monoid and $N$ be a submonoid of the additive monoid $\mathbf{R}_{+}$. By a $N$-valued divisor theory for $S$ we mean a monoid homomorphism $\varphi$ of $S$ into a direct sum $N^{(I)}$ such that $x \leq y$ in $S$, for $x, y \in S$, is equivalent to $\varphi(x) \leq \varphi(y)$ in $N^{(I)}$ and such that each element of $N^{(I)}$ is the pointwise minimum in $N^{(I)}$ of finitely many elements from $\varphi(S)$.

Corollary 2. Let $S$ be a monoid of finite adapted character such that (after some normalization) all states on $(G, S)$ belonging to the defining family are of the same range, say $M$. Then for some subset $I$ of essential states, the mapping $\varphi: S \rightarrow\left(M \cap \mathbb{R}_{+}\right)^{(I)}$ defined by $\varphi(x)(i)=i(x)$ is a $M \cap \mathbf{R}_{+}$-valued divisor theory for $S$.

Proof. As in the proof of the theorem choose a subset $I$ of essential states such that for $i, j \in I$ with $i \neq j$ also $i \neq r j$ for all $r>0$. Consider for given $i \in I, m \in M \cap \mathbf{R}_{+}$the following element in $\left(M \cap \mathbf{R}_{+}\right)^{(I)}$

$$
\varepsilon(j)= \begin{cases}m, & j=i \\ 0, & j \neq i\end{cases}
$$

By assumption, there exists $y_{i} \in G$ with $i\left(y_{i}\right)=m$. Since $i$ is essential there exists some $x_{i} \in S$ with $i\left(x_{i}\right)=m . S$ being of finite character, by part (1) of the lemma the set $J=\left\{j \in I \mid j\left(x_{i}\right) \neq 0\right\}$ is finite. For $j \in J, j \neq i$, by step (1) in the proof of the lemma we cannot have $S \cap j^{-1}(0) \subset i^{-1}\left(\mathbf{R}_{-}\right)$. That is, there exists some $y_{j} \in S$ with $j\left(y_{j}\right)=0$ and $i\left(y_{j}\right)>0$. Choose $n_{j} \in \mathbb{N}$ such that $i\left(n_{j} y_{j}\right)>m$ and let $x=n_{j} y_{j}$ for $j \in J, j \neq i$. Thus we obtain

$$
\min _{k \in J \cup\{i\}} j\left(x_{k}\right)= \begin{cases}m, & j=i \\ 0, & j \in J \backslash\{i\} \\ 0, & j \notin J\end{cases}
$$

and hence $\varepsilon(j)=\min _{k \in J \cup\{i\}} \varphi\left(x_{k}\right)(j)$ for all $j \in I$. That is, $\varepsilon$ is the minimum in $\left(M \cap \mathbf{R}_{+}\right)^{(I)}$ of finitely many elements from $\varphi(S)$. Finally, any element of $\left(M \cap R_{+}\right)^{(I)}$ is a finite sum of such elements $\varepsilon$ and any finite sum of elements of the form $\min _{k \in J_{i}} \varphi\left(x_{k}\right)$ is again of this form. Thus, every element of $(M \cap \mathbb{R})^{(I)}$ is the minimum in $(M \cap \mathbf{R})^{(I)}$ of finitely many elements from $\varphi(S)$. The remaining conditions for a divisor theory are obviously satisfied. Q.E.D.

This corollary applies not only to algebraic objects like Krull monoids but also to the polyhedral cones of convex analysis. 
Example. Let $S$ be a polyhedral cone in $\mathbb{R}^{n}$, i.e., $S$ is the intersection of finitely many homogeneous halfspaces $f_{i}^{-1}\left(\mathbf{R}_{+}\right), i=1, \ldots, m$. To simplify, assume the interior of $S$ to be nonempty and hence $G=S-S=\mathbf{R}^{n}$. Obviously, with addition $S$ is a monoid of finite adapted character and the range of the linear functional $f_{i} \neq 0$ is equal to $\mathbf{R}$ for each $i$. The essential states in this case, say $f_{1}, \ldots, f_{k}$ without restriction, correspond to those hyperplanes determined by a facet, i.e., $f_{i}^{-1}(0) \cap S$ is an $(n-1)$-dimensional face of $S, i=1, \ldots, k$. The $\mathbb{R}_{+}$-valued divisor theory for $S$ as constructed in the corollary is the mapping $\varphi: S \rightarrow \mathbf{R}_{+}^{k}$ with the $i$ th component of $\varphi(x)$ given by the evaluation of $f_{i}$ at $x$. The divisor theory may be viewed as a minimal representation of the preorder defined by $S$ in terms of the standard componentwise ordering. The ice cream cone discussed earlier (Example (3)) does not admit any divisor theory.

\section{KRULL MONOIDS AND KRULL DOMAINS}

A common description of a Krull domain is that of an integral domain which is defined by a family of finite character consisting of rank-one discrete valuations ([3, p. 190], [7, p. 86]). As remarked already, the multiplicative monoid of a Krull domain must be a Krull monoid. It is interesting that this property, which employs only the multiplicative operation, already characterizes Krull domains among integral domains. This is a consequence of the result obtained in the previous section saying that a Krull monoid may be defined also by the family of its essential states. Let $R$ be an integral domain with quotient field $K, R^{*}=R \backslash\{0\}$ its multiplicative monoid with quotient group $K^{*}=K \backslash\{0\}$.

Proposition. An integral domain is a Krull domain (rational generalized Krull domain) if and only if its multiplicative monoid is a Krull monoid (rational generalized Krull monoid).

Proof. It suffices to show that the integral domain $R$ must be a Krull domain if $R^{*}$ is a Krull monoid. The same argument then applies with rational states instead of discrete states. Let $R^{*}$ be a Krull monoid with a defining family $F$ which, by Corollary 1, is of finite character and consists of discrete essential states on $\left(K^{*}, R^{*}\right)$. For $f \in F$ and $x, y \in K^{*}$ with $x+y \in K^{*}$ by the definition of an essential state there exist $u, v \in R^{*}$ and $z \in K^{*}$ such that

$$
z=u x^{-1}, z=v y^{-1} \quad \text { and } \quad f(z)=\max \left\{f\left(x^{-1}\right), f\left(y^{-1}\right)\right\} .
$$

Hence

$$
f\left((x+y)^{-1}\right)=f\left(z(u+v)^{-1}\right)=f(z)-f(u+v) \leq f(z)
$$

and

$$
f(x+y)=-f\left((x+y)^{-1}\right) \geq-f(z)=\min \{f(x), f(y)\} .
$$

Defining $\bar{f}: K \rightarrow \mathbb{R}$ by $\bar{f}(x)=f(x)$ for $x \in K^{*}$ and $\bar{f}(0)=+\infty$, we obtain a valuation $\bar{f}$ on $K$. The family $\bar{F}=\{\bar{f} \mid f \in F\}$ of discrete valuations on $K$ is of finite character and $\bar{F}$ defines $R$ because $F$ defines $R^{*}$. Q.E.D. 
We conclude with some remarks concerning the proposition and its implications.

Remarks. (1) The above proof shows that for an essential state $f$ on the multiplicative monoid of an integral domain the set $P=f^{-1}\left(\mathbb{R}_{+}\right)$is not only a monoid but a subring, actually an essential rank-one valuation ring. One shows similarly, for an integral domain $R$ with quotient field $K$, that more general the valuation rings of $K$ which are essential for $R$ can be characterized multiplicatively as the sets $P \cup\{0\}$ with $P \subset K^{*}$ such that $P P \subset P, P \cup P^{-1}=K^{*}$ and $P$ equal to the monoid of fractions of $R^{*}$ with respect to $R^{*} \cap P \cap P^{-1}$.

(2) The above remark can be used to make counterexamples from Krull ring theory applicable to Krull monoids. In [5, p. 84] an integral domain $R$ defined by a family of finite real character is constructed which cannot be defined by a family consisting of essential valuations only. (This answers a question of Krull in the negative.) Hence $R^{*}$ is a monoid of finite character which cannot be defined by a family of essential states, because the latter would be necessarily essential valuations. Thus this example of Griffin shows that in the theorem of $\S 2$ the addendum "adapted" cannot simply be omitted.

(3) The multiplicative monoids of Krull domains are particular examples of Krull monoids. Moreover, it is easily verified that any submonoid $S$ of the multiplicative monoid $R^{*}$ of a Krull domain $R$ is a Krull monoid, provided $1 \in S$ and two elements from $S$ divide each other in $S$ if they divide in $R$. Actually, in this way one may arrive at Krull monoids $S$ which cannot be "realized" as the multiplicative monoid of some Krull domain. Consider for example in an arbitrary Krull domain $R$ some nonunit $a \neq 0$ and let $S=\left\{a^{n} \mid n=0,1,2, \ldots\right\}$. Since two elements from $S$ divide in $S$ if they divide in $R, S$ must be a Krull monoid. Suppose $S$ could be realized as the multiplicative monoid $T^{*}$ of some integral domain $(T, \cdot,+)$. (Whereas the multiplicaton "." coincides with that of $R$ on $S$, the addition "+" may be different from that in $R$.) If in $T \quad 1+a=0$, then $a=-1$ and $a^{2}=(-1)^{2}=1$. This is impossible since $a$ is not a unit in $R$. Hence $1+a \neq 0$ and $1+a \in T^{*}=$ $S$. Therefore $1+a=a^{n}$ for some $n \geq 1$ and $1=a^{n}-a=a\left(a^{n-1}-1\right)$. In particular, $a^{n-1}-1 \in T^{*}=S$ and $a$ is a unit in $R$, which is impossible. Thus $S$ cannot be realized as the multiplicative monoids of some integral domain.

(4) Call a property of an integral domain $R$ purely multiplicative whenever any integral domain $T$ with multiplicative monoid $T^{*}$ isomorphic to $R^{*}$ has this same property too. By the proposition, being a Krull domain is a purely multiplicative property among integral domains. This nice feature of a Krull domain, which seems not to be stated in the literature, is obscured by the commonly adopted definitions of a Krull domain. (Simple examples show that being a Dedekind domain or a Prüfer domain, respectively, is not a purely multiplicative property among integral domains.) In order to make the purely multiplicative character clear right from the beginning, it would be preferable to define a Krull domain as an integral domain for which the multiplicative monoid is a 
Krull monoid. Corollaries 1 and 2 then supply further information, also about Krull domains and rational generalized Krull domains, respectively.

\section{ACKNOWLEDGMENT}

The author wishes to thank the referee for helpful comments.

\section{REFERENCES}

1. N. Bourbaki, Commutative algebra, Addison-Wesley, Reading, Mass., 1972.

2. L. G. Chouinard II, Krull semigroups and divisor class groups, Canad. J. Math. 33 (1981), 1459-1468.

3. R. Gilmer, Commutative semigroup rings, The University of Chicago Press, Chicago and London, 1984.

4. K. R. Goodearl, Partially ordered Abelian groups with interpolation, American Mathematical Society, Providence, 1986.

5. M. Griffin, Families of finite character and essential valuations, Trans. Amer. Math. Soc. 130 (1968), 75-85.

6. E. Grosswald, Topics from the theory of numbers, Macmillan, New York, 1966.

7. H. Matsumura, Commutative ring theory, Cambridge University Press, Cambridge, 1986.

FB Mathematik, Universität Bremen, 28 Bremen, West Germany 